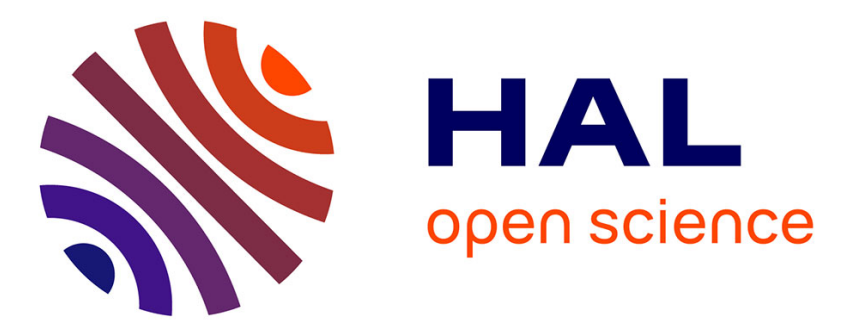

\title{
Precoating for improving the cleaning of filter media clogged with metallic nanoparticles
}

\author{
Nassim Khirouni, Augustin Charvet, Clara Drisket, Alain Ginestet, \\ Dominique Thomas, Denis Bémer
}

\section{- To cite this version:}

Nassim Khirouni, Augustin Charvet, Clara Drisket, Alain Ginestet, Dominique Thomas, et al.. Precoating for improving the cleaning of filter media clogged with metallic nanoparticles. Process Safety and Environmental Protection, 2021, 147, pp.311-319. 10.1016/j.psep.2020.09.045 . hal-02995313

\section{HAL Id: hal-02995313 \\ https://hal.science/hal-02995313}

Submitted on 9 Nov 2020

HAL is a multi-disciplinary open access archive for the deposit and dissemination of scientific research documents, whether they are published or not. The documents may come from teaching and research institutions in France or abroad, or from public or private research centers.
L'archive ouverte pluridisciplinaire HAL, est destinée au dépôt et à la diffusion de documents scientifiques de niveau recherche, publiés ou non, émanant des établissements d'enseignement et de recherche français ou étrangers, des laboratoires publics ou privés. 


\title{
Precoating for improving the cleaning of filter media clogged with metallic nanoparticles
}

\author{
KHIROUNI N. ${ }^{1,2}$, CHARVET A..$^{*}$, DRISKET C. ${ }^{2}$, GINESTET A. ${ }^{3}$,THOMAS D. ${ }^{2}$, BEMER D. ${ }^{1}$
}

\author{
${ }^{1}$ Institut National de Recherche et de Sécurité (INRS), F-54519 Vandœuvre-lès-Nancy, France \\ ${ }^{2}$ Université de Lorraine, CNRS, LRGP, F-54000 Nancy, France \\ ${ }^{3}$ Centre Technique des Industries Aérauliques et Thermiques (CETIAT), F-69603 Villeurbanne, France \\ *Corresponding author \\ E-mail: augustin.charvet@univ-lorraine.fr
}

\begin{abstract}
Filtration of dust nanoparticles emitted from metallurgical processes is currently problematic, due to a rapid increase of the pressure drop and an inefficient filter cleaning, inducing high maintenance costs. This paper investigates the use of precoating technique to overcome the cleaning difficulties caused by the metallic nanoparticles. It consists of protecting the filter surface with a dust layer composed of micron-sized particles that is easily discharged upon cleaning. In this study, the pulse-jet cleaning of flat filters with and without precoating is evaluated. The aim is to determine the effect of operating conditions on the cleaning efficiency and provide guidelines on the application of precoating. Different precoating materials are tested, to determine whether the material choice is of influence. Experimental results highlighted the increase of the collection efficiency and dust holding capacity using precoating. The cleaning efficiency was significantly improved from $10 \%$ without precoating to $90 \%$ with precoating. Clogging/unclogging cycles showed that the use of precoating allowed stabilizing the filtration process.
\end{abstract}

Keywords: Filtration, metallic nanoparticles, cleaning efficiency, filter media, precoating

\section{Introduction}

Fibrous media are widely used in the industry for dust emissions control. During filtration, a dust cake will form on the filter surface that would increase the resistance to airflow. Therefore, continuous filtration process over time is achieved by a frequent cleaning of the loaded filter elements. Filter cleaning is generally performed by injecting short bursts of compressed air in the reverse direction of filtration. Two operation modes may be implemented: (i) a cleaning on time, for which the filter is cleaned periodically at a fixed time interval, or (ii) a cleaning on demand for which the filter cleaning is activated once reaching a limit value of the pressure drop that depends on the ventilation capacity. It was shown that the clean on time mode is more convenient for pleated filter cartridges with high pleat number (Lo et al., 2010). Incomplete removal of the dust cake from the filter surface will result in an unstable filtration cycles leading to a rapid increase of the pressure drop and a significant decrease of the dust holding capacity. This implies that the filter elements need to be replaced, which can be very expensive. In the particular case of metallic ultrafine particles emitted from metallurgical processes such as thermal spraying, the clogging of the filter media seems to be irreversible (continuous increase of the residual pressure drop after cleaning). In a previous work (Khirouni et al., 2020), the cleaning efficiency of filters clogged with these metallic nanoparticles was evaluated. Results have revealed a patchy inefficient cleaning that does not exceed $30 \%$ according to the removed mass. The metallic nanostructured filter cake tends to have a high adhesion force that makes its detachment difficult. This is a source of real concern because the filters life span is directly linked to the regeneration efficiency. Inefficient cleaning

KHIROUNI N., CHARVET A.,DRISKET C., GINESTET A., THOMAS D., BEMER D., Precoating for improving the cleaning of filter media clogged with metallic nanoparticles, Process Safety and Environmental Protection, 147 (2021) $311-319$ (10.1016/j.psep.2020.09.045) 
means that the filters cannot be used for a long period of time, inducing high maintenance costs.

One of the solutions that are recommended to overcome the cleaning difficulties caused by sticky adhesive dusts is the use of precoating (Peukert and Wadenpohl, 2001). This technique, often viewed as a low cost and practical solution, consists of protecting the filter surface with a dust layer composed of micron-sized particles, that would be easily discharged upon cleaning, on which the ultrafine particles are collected. The major drawback is the need to reapply the precoating layer after each cleaning cycle, thus continuous filtration process can be ensured by parallel filtration chambers (Schmidt, 2004). This protective layer would serve as a granular bed for the capture of nanoparticles, enhancing by that the collection efficiency and limiting the clogging of the filter media. Förster et al. (2016) gave a particular attention to the collection efficiency of the precoat layer for the capture of copper nano-aerosol in the size range of 5 and $40 \mathrm{~nm}$. The precoat layer was composed of alumina particles with a mean diameter of $4 \mu \mathrm{m}$. Results showed an increase of the collection efficiency with the cake thickness. However, increasing the face velocity has resulted in a decrease of the collection efficiency. As the governing collection mechanism for nanoparticles is Brownian diffusion, an increase of the velocity will cause a decrease of the particles residence time. The authors also highlighted that higher temperatures are associated with an increase of the particles collection due to an increase of the diffusion coefficient.

The precoating material must be chemically inert, non-toxic and of low cost. An important selection criterion reported in the literature is the precoating particle size distribution. Too coarse particles will cause the formation of unwanted deposits in the ducts and a non-uniform layer, whereas too fine particles could be associated with an inefficient cleaning of the filter media. As for the layer thickness, a minimum of $1 \mathrm{~mm}$ is recommended (Schmidt and Löffler, 1990). According to the used material, this protective layer could also be employed for humidity adsorption or as a flame retardant (Schmidt and Pilz, 1996). Hajar et al. (2015) added an intriguing twist to the precoating material, by considering a mix of precoating powder with activated carbon. This mix gives the precoating layer an additional advantage, consisting in the adsorptive capacity of the activated carbon for eliminating organic compounds or heavy metals from the gas stream.

The implementation of this precoating technique has been reported in the literature, but the available data are not sufficient for operating filtration systems. Bémer et al. (2013) investigated the use of precoating for improving the regeneration of a pleated filter cartridge clogged with metallic ultrafine particles. The authors applied a deposited mass of 24 g.m $\mathrm{m}^{-2}$ of limestone dust with a mass median diameter of 3.6 $\mu \mathrm{m}$. Results showed that the use of precoating allows obtaining stable filtration cycles. (Schiller and Schmid, 2014; Schiller and Schmid, 2015) provided experimental data on the application of precoating for the filtration of ultrafine particles emitted from biomass burners. Different precoating materials (limestone, hydrated limestone and $\mathrm{SiO}_{2}$ with a median number diameter $<1 \mu \mathrm{m}$ ) and filter media were tested. The authors used the combination of filter and precoating that would have the lowest pressure drop. The results obtained using a bag filter did show that the use of precoating resulted in a stable filtration process. The authors also explored the possibility of reusing the precoating up to three times, which allows savings up to $67 \%$ in precoating mass. Despite the reported articles, many questions are still unanswered when it comes to the application of precoating in practice. The first being the necessary mass of precoating to be applied and its effect on the cleaning efficiency. No available results describe the variation of the cleaning efficiency with the precoating thickness. Although a $1 \mathrm{~mm}$ thickness is recommended in the literature, this information is not fully practical in reality as it would be difficult to assess. From an industrial point of view, it is preferable to use the minimum quantity possible. Furthermore, the influence of the operating conditions while applying precoating is unknown, an information that is valuable for optimizing conditions and costs.

This paper investigates the use of precoating for improving the pulse-jet cleaning of flat filters clogged with metallic nanoparticles. The aim is to validate the feasibility of precoating to overcome the cleaning difficulties and provide guidelines on the application of this technique. Although the acquired results with flat filters cannot be directly applied to full scale filtration systems due to geometric and flow differences, they are of interest to identify patterns that may help reduce operating costs and make the precoating more appealing for an industrial application. To this end, the influence of the deposited mass of precoating, the cleaning air pressure and the filtration velocity is studied. The effect of these parameters is extremely important because they can be easily adjusted and controlled in the industry. A special focus is also paid to the precoating material. The influence of the precoating 
material on the cleaning efficiency was not studied in the literature, an information that is of importance when choosing the precoating. Commercially available products and a metallic powder recovered in the hopper of a cyclone placed upstream of the filtration device are tested. The use of the metallic powder as precoating could allow making savings as the powder is available on site and generally disposed of as waste.

\section{Materials and methods}

\subsection{Experimental pilot}

A pilot thermal spraying process, using electric arc, allows obtaining dust emissions similar to those met in industrial applications. As shown in figure 1, the electric arc gun (M25 Margarido ${ }^{\circledR}$ ) is fed by zinc wires (of $2 \mathrm{~mm}$ diameter) and the melted metal is sprayed on a rotating cylinder by compressed air. The generated fumes are sucked towards a protection dust collection system. A fraction of the fumes is directed to the studied filtration system for conducting experiments on flat filters of $14 \mathrm{~cm}$ diameter. The generated aerosol was characterized in a previous work (Bémer et al., 2013), results revealed the presence of a micronic fraction with a median mass diameter of $9 \mu \mathrm{m}$. In order to eliminate this micronic fraction, a cyclone (URG-2000-30EC ${ }^{\circledR}$ ) with a cut diameter of $2.5 \mu \mathrm{m}$ is placed ahead of the filter holder. The size distribution measurement of the metallic ultrafine particles, conducted downstream of the cyclone using a scanning mobility particle sizer (5.403 Grimm ${ }^{\circledR}$ ), after $1 / 100$ dilution (VKL 100, Palas $\left.{ }^{\circledR}\right)$, revealed a high number concentration of about $210^{8}$ particules. $\mathrm{cm}^{-3}$ with a mean mobility equivalent diameter of $70 \mathrm{~nm}$. The dust laden air is sucked through the flat filter by a pump and the flow is controlled by a sonic nozzle. The tested filter media is a corrugated cellulose fibre medium with a nanofiber layer, typically used for the filtration of metallurgical dusts, its main characteristics are given in table 1 . The precoating is applied using a brush powder dispersion generator (RBG 1000, PALAS $^{\circledR}$ ) prior to the filtration of the metallic ultrafine particles. This is controlled by the valves 1 and 2 . The generated precoating is sampled rather than being directly injected to the filter surface in order to avoid undesired deposits in the ducts and to ensure the application of a uniform dust cake. Indeed, coarse particles tend to settle in the canalization by sedimentation due to their size. Different precoating quantities are applied by controlling the precoat layer pressure drop using a pressure sensor (Keller $\left.{ }^{\circledR}\right)$. The deposited mass is then determined by weighing the filter before and after loading. Once the precoating is applied, the metallic ultrafine particles are filtered until reaching a limit value of the pressure drop, followed by filter cleaning.

The pulse-jet cleaning of the flat filters, with and without precoating, is performed using an injection nozzle of $3 \mathrm{~mm}$ diameter and a pulse duration of 0.2 $\mathrm{s}$. The pulse-jet injection is controlled by an electrovalve $\left(230 \mathrm{~V} \mathrm{ac}, \mathrm{RSPRO}^{\circledR}\right)$. The cleaning air pressure was varied between 2 and 4 bar. The cleaning is triggered once reaching a maximum pressure drop value that was set at different values (between 500 and $2000 \mathrm{~Pa}$ ) to investigate the effect of the collected mass of metallic nanoparticles. The cleaning efficiency of the flat filters is evaluated according to the removed mass, expressed as follows:

$$
\mathrm{R}_{\mathrm{M}}=\frac{\text { Removed mass }}{\text { Initial cake mass }}
$$

The removed mass is evaluated by weighing the filter before and after cleaning. The cleaning efficiency according to the residual pressure drop is not considered in this study as it was found to be insufficient to describe the regeneration of the filter surface in a previous work (Khirouni et al., 2020). Indeed, in the case of a patchy cleaning the formed holes in the filter cake will constitute preferential paths for the airflow causing by that a considerable decrease of the residual pressure drop. This gives rise to high cleaning efficiencies that do not reflect the amount of dust still adhering to the surface.

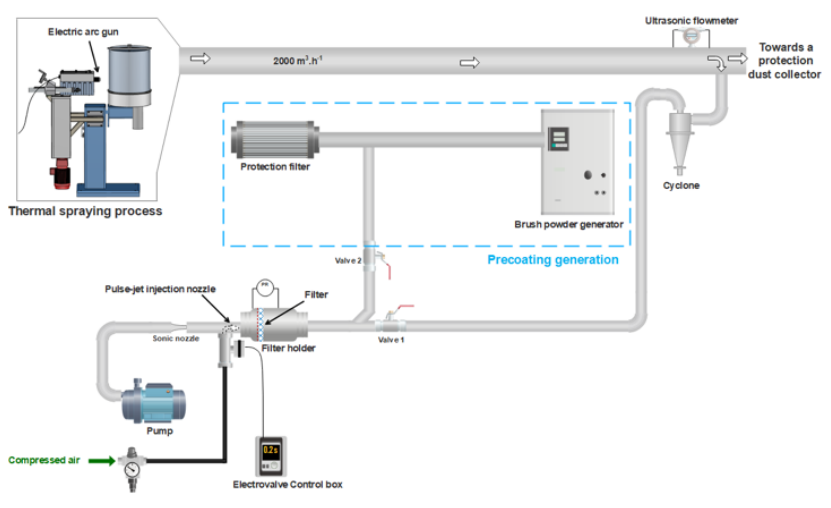

Figure 1: Experimental pilot

In order to give an insight whether the precoating choice is of importance or not, different powders are used. The main characteristics of the different precoating materials tested are given in table 2 . The materials $\mathrm{P} 1$ and $\mathrm{P} 2$ are typically commercialized and known to be used in the industry for filter conditioning. P3 represents the micron-sized dust particles recovered in the hopper of the filtration system's cyclone, which consist of a metallic powder. 
The number size distributions of the particles of the generated precoating materials, measured with an Aerodynamic Particle Sizer (Model 3321, $\mathrm{TSI}^{\circledR}$ ), are given in figure 2 . For the three materials, the median diameter is around $1 \mu \mathrm{m}$. It can be noticed that for the P3 powder the size distribution is bimodal, with a small particle mode below $1 \mu \mathrm{m}$ and another around $3 \mu \mathrm{m}$.

Table 1: Characteristics of the filter medium.

\begin{tabular}{|c|c|}
\hline Composition & Cellulose \\
\hline Surface treatment & Nanofibre layer \\
\hline $\begin{array}{l}\text { Average fibre diameter-Davies } \\
(\mu \mathrm{m})\end{array}$ & $23 \pm 1$ \\
\hline Mean nanofibers diameter $(\mathrm{nm})^{1}$ & $212 \pm 74$ \\
\hline Grammage (g.m ${ }^{-2}$ ) & $138 \pm 2$ \\
\hline Thickness $(\mathrm{mm})^{2}$ & $0.28 \pm 0.02$ \\
\hline Porosity ${ }^{3}$ & $0.70 \pm 0.06$ \\
\hline Permeability $\left(\mathrm{m}^{2}\right)$ & $3.62 \pm 0.1810^{-11}$ \\
\hline \multicolumn{2}{|l|}{${ }^{1}$ Measurement by SEM image analysis } \\
\hline \multicolumn{2}{|l|}{${ }^{2}$ Measurement by micrometer screw } \\
\hline${ }^{3}$ Measurement by pycnometry & \\
\hline
\end{tabular}

Table 2: Characteristics of the used precoating materials

\begin{tabular}{cccc}
\hline Precoating & Composition & $\begin{array}{l}\text { Density } \\
\left(\mathbf{k g} \cdot \mathbf{m}^{-3}\right)\end{array}$ & $\begin{array}{l}\text { Number median } \\
\text { diameter of the } \\
\text { generated } \\
\text { aerosol }(\boldsymbol{\mu m})\end{array}$ \\
\hline P1 & $\mathrm{SiO}_{2}$ & 2001 & 0.88 \\
\hline P2 & $\mathrm{CaCO}_{3}$ & 2669 & 0.95 \\
\hline P3 & $\mathrm{Zn}$ & 5093 & 1.02 \\
\hline
\end{tabular}

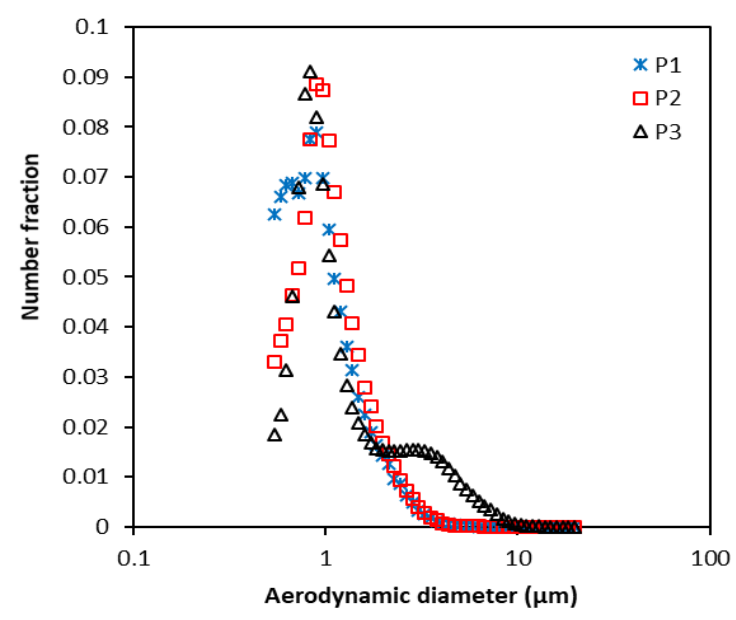

Figure 2: Particles number size distribution of the tested precoating materials

\subsection{Precoat layer collection efficiency}

In order to determine the initial filtration efficiency of the precoating layer for the collection of nanoparticles an experimental setup was developed, given in figure 3 . The precoating is applied using the brush generator (RBG 1000, PALAS $^{\circledR}$ ) on a high efficiency glass microfiber filter of $60 \mathrm{~mm}$ diameter. This type of filter was used to avoid depth filtration and obtain an accurate estimation of the precoating collection efficiency. Once the precoating layer is formed, the collection efficiency of graphite nanostructured particles, generated with a spark generator (GFG 1000, PALAS $^{\circledR}$ ), is measured using an electrostatic classifier (Model 3082, TSI $^{\circledR}$ ) coupled with a condensation particle counter (Model 3776, $\mathrm{TSI}^{\circledR}$ ). The graphite nanostructured particles size distribution presents a median mobility equivalent diameter close to $40 \mathrm{~nm}$. The fractional filtration efficiency $\left(E_{i}\right)$ is determined with the following expression:

$$
\mathrm{E}_{\mathrm{i}}=1-\frac{\mathrm{C}_{\text {out }, \mathrm{i}}}{\mathrm{C}_{\mathrm{in}, \mathrm{i}}}
$$

With $\mathrm{C}_{\mathrm{in}, \mathrm{i}}$ and $\mathrm{C}_{\text {out,i }}$ the upstream and downstream fractional number concentrations respectively, i stands for the different particle sizes.

After measuring the clean filter efficiency $\left(E_{\text {filter,i }}\right)$ and the "filter + precoating" efficiency $\left(\mathrm{E}_{(\mathrm{filter}+\text { precoating), }}\right)$, the precoating layer collection efficiency alone $\left(E_{\text {precoating, }}\right)$ is calculated as follows by assuming two filters in series:

$$
\mathrm{E}_{\text {precoating,i }}=1-\frac{1-\mathrm{E}_{(\text {filter+precoating) } \mathrm{i}}}{1-\mathrm{E}_{\mathrm{filter}, \mathrm{i}}}
$$

The collection efficiency is determined for different precoating masses with a face velocity of $2.5 \mathrm{~cm} . \mathrm{s}^{-1}$. The deposited mass of precoating is determined by weighing the filter before and after loading.

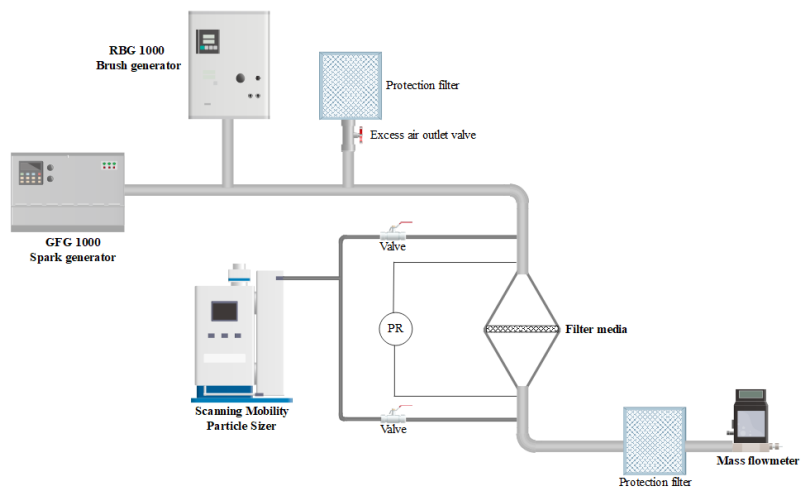

Figure 3: Schematic diagram of the used setup for measuring the precoat layer initial collection efficiency 


\section{Results and discussion}

\subsection{Effect of precoating on the filtration} performances

The variation of the precoating layer initial collection efficiency with the precoating mass per unit area is given in figure $4(a, b, c)$. Increasing the deposited mass, i.e. the cake thickness, allows enhancing the collection efficiency to surpass 95\% whatever the precoating material. Indeed, the thicker the precoating layer is the more probable that the nanoparticles get collected in the cake depth. These results show that the precoating layer acts as a granular bed where the nanoparticles are captured by the precoating particles, noting that the governing collection mechanism in this size range is Brownian diffusion. This is interesting because it implies that when applying an appropriate amount of precoating the initial filtration efficiency of the (filter + precoating) will be close to the unity. The necessary deposited mass of precoating to reach a high efficiency varies with the precoating material. This can be attributed to the differences in the cake structure and porosity associated with the different materials. In industrial applications, often a concentration of precoating between 25 and 50 g.m ${ }^{-2}$ is applied. Such an order of magnitude clearly allows ensuring high filtration efficiency.

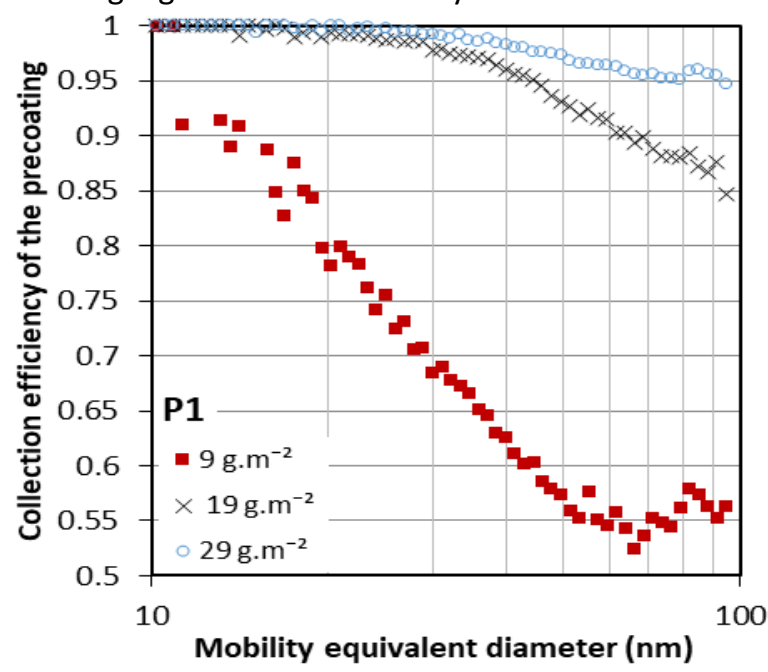

Figure 4.a: Evolution of the initial collection efficiency of the precoating layer with the deposited mass per unit area using precoating material P1 for a face velocity of $2.5 \mathrm{~cm} . \mathrm{s}^{-1}$

Clogging experiments of flat filters with metallic nanoparticles, generated with the experimental pilot presented in figure 1 , were performed with and without precoating. The results given in figure $5(a, b$, c) show the same trend for the three materials. The increase of the precoating thickness is associated with a decrease of the pressure drop evolution.

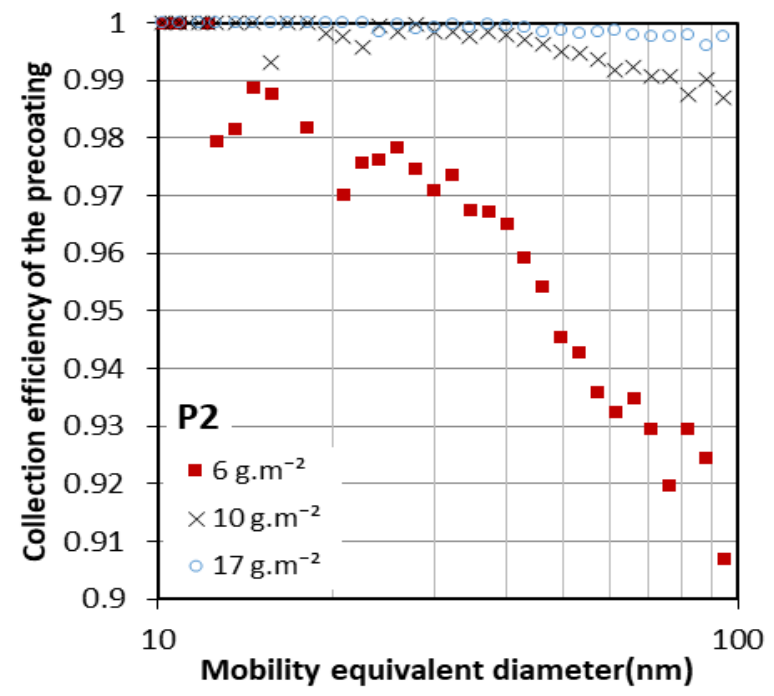

Figure 4.b: Evolution of the initial collection efficiency of the precoating layer with the deposited mass per unit area using precoating material $\mathrm{P} 2$ for a face velocity of $2.5 \mathrm{~cm} . \mathrm{s}^{-1}$

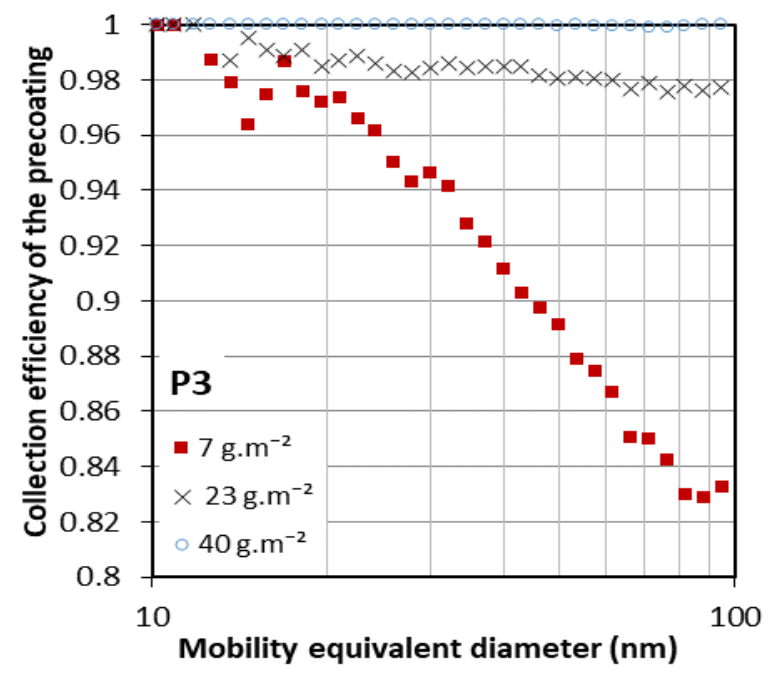

Figure 4.c: Evolution of the initial collection efficiency of the precoating layer with the deposited mass per unit area using precoating material P3 for a face velocity of $2.5 \mathrm{~cm} . \mathrm{s}^{-1}$

Indeed, the precoating layer constitutes an additional filtering medium for the collection of ultrafine particles allowing to increase the dust holding capacity, which is extremely interesting at an industrial scale. It can be observed that for the same final pressure drop the collected mass of nanoparticles could be raised up to three times compared to that without precoating. The same trend 
was observed by Charvet et al. (Charvet et al., 2018) when studying the association of fibrous media. The authors registered a decrease of the resistance when placing a medium filter (composed of microfibers) upstream of a HEPA filter. This allowed favouring depth filtration in the medium filter and delaying surface filtration in the HEPA filter, thus decreasing the resistance to airflow and increasing the dust holding capacity. With a precoating layer, nanoparticles are collected in the depth. Therefore, with increasing the precoating layer thickness the dust holding capacity increases and the protective effect of precoating is promoted as the probability for nanoparticles to reach the filter surface is reduced. However, when the precoating layer is too thin, as the one with a deposited mass of $5 \mathrm{~g} . \mathrm{m}^{-2}$ of P2 material, nanoparticles will reach the filter surface and the pressure drop evolution is close to the one without precoating. Using a suitable thickness, nanoparticles are distributed in a more important volume, inducing a decrease of the pressure drop evolution and a lower cake resistance. This may explain the differences of the pressure drop evolution slopes with increasing the precoating thickness. Besides, the mixtures of precoating and ultrafine particles would obviously present different porosities. Further increasing the precoating thickness is consequently not necessary as the layer already has a collection efficiency close to one.

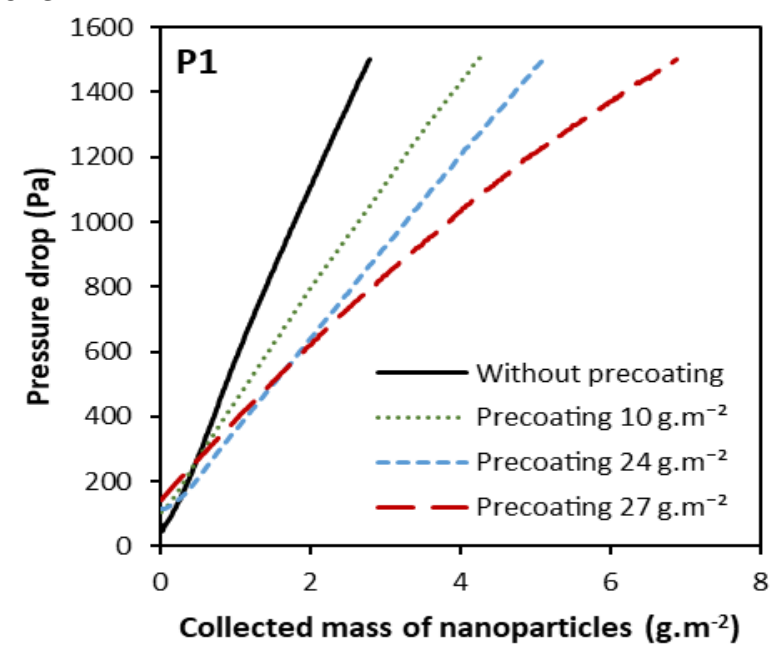

Figure 5.a: Effect of precoating on the evolution of the pressure drop during clogging with metallic nanoparticles using material P1 for a face velocity of $3 \mathrm{~cm} \cdot \mathrm{s}^{-1}$

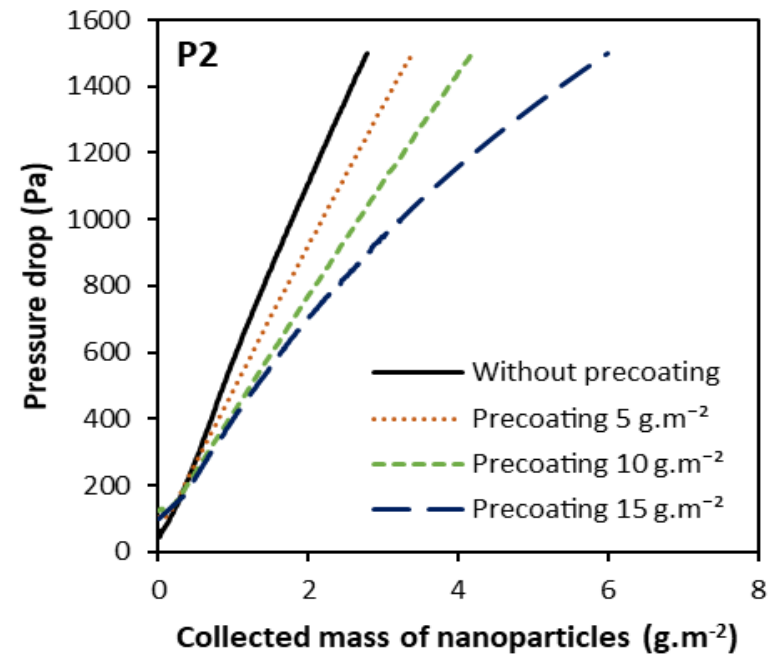

Figure 5.b: Effect of precoating on the evolution of the pressure drop during clogging with metallic nanoparticles using material P2 for a face velocity of $3 \mathrm{~cm} . \mathrm{s}^{-1}$

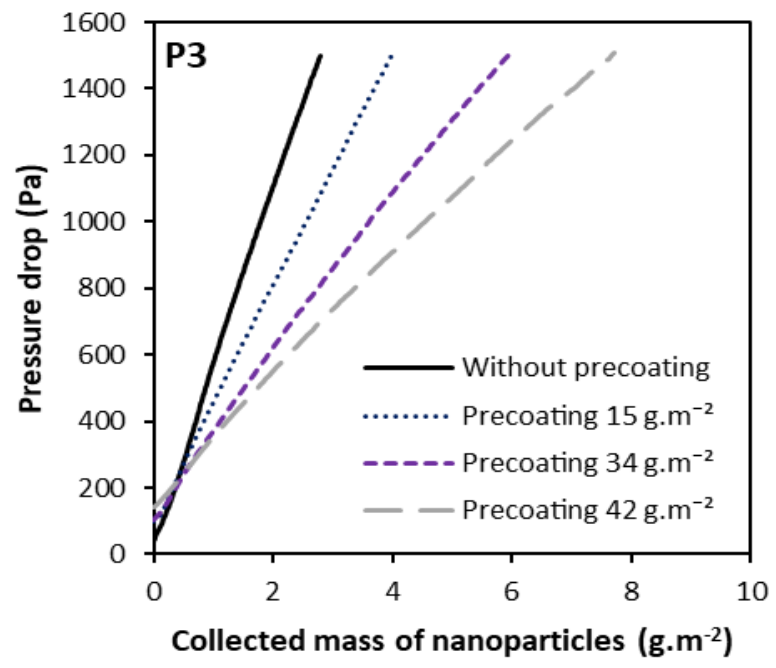

Figure 5.c: Effect of precoating on the evolution of the pressure drop during clogging with metallic nanoparticles using material P3 for a face velocity of $3 \mathrm{~cm} . \mathrm{s}^{-1}$

\subsection{Improving the cleaning efficiency with precoating}

Figure 6 gives the pressure drop of the precoating layer alone (after subtracting the clean filter pressure drop) as a function of the deposited mass for the three different materials, registered prior to the filtration of the metallic nanoparticles. The material P3 has the lowest pressure drop evolution, this might be due to the bimodal size distribution that presents 
a large particle mode of about $3 \mu \mathrm{m}$. As for the other materials, differences of the particles shapes and densities could be behind the observed disparity.

Figures 7, 8 and 9 summarize the influence of precoating thickness, maximum pressure drop value before cleaning and cleaning air pressure on the cleaning efficiency using the different materials. The maximum pressure drop before cleaning was set at different values to investigate the effect of the collected mass of metallic nanoparticles, which can be deduced from figure 5 . The chosen values of the maximum pressure drop before cleaning (500-1500 $\mathrm{Pa}$ ) are close to the ones used in industrial filtration systems. The cleaning was performed using two values of the air cleaning pressure: 2 and 4 bar. The ability to reduce the air cleaning pressure, without affecting the cleaning efficiency, is of interest at an industrial scale as it could help reduce compressed air consumption. Experimental data reveal that the increase of the precoating thickness allows improving the cleaning efficiency. Results show that thin layers, as the one with a deposited mass of $10 \mathrm{~g} \cdot \mathrm{m}^{-2}$ of P3 material, are not sufficient to provide full protection of the filter surface. The precoating must be thick enough to prevent the nanoparticles from penetrating to the filter surface. These findings are in agreement with the precoating layer initial collection efficiency measurements (figure 4). A precoating thickness that has a collection efficiency close to one would be suitable to ensure efficient cleaning. Indeed, it would prevent the nanoparticles from reaching the filter surface. Comparing to the results without precoating, a significant improvement of the cleaning efficiency is observed, going from roughly $15 \%$ to $90 \%$ with precoating. The collected mass of nanoparticles has a beneficial effect on the cleaning efficiency. This is due to the increase of the cohesion forces between the particles in the dust cake. Zhang and Schmidt (2012) observed similar results when applying "post-coating" which consisted of filtering finer particles on top of a dust cake composed of coarser particles. The nanoparticles would increase Van der Waals forces between particles in the precoating layer, raising by that the cohesion forces and the cake resistance which facilitate its discharge. The results obtained with the precoating material P3 are very promising taking into consideration that this powder is recovered from the cyclone hopper. The precoating material $\mathrm{P} 2$ has the advantage of requiring a lower deposited mass for obtaining an efficient cleaning, but is associated with a higher pressure drop. This highlights the influence of the used material, as the necessary mass differs due to differences in the cake structure and porosity. Results also indicate that in order to reduce the cleaning air pressure, the used mass of precoating must be increased a bit, as it would help to increase cohesion forces (Sievert and Löffler, 1987) (Clift and Seville, 1993).

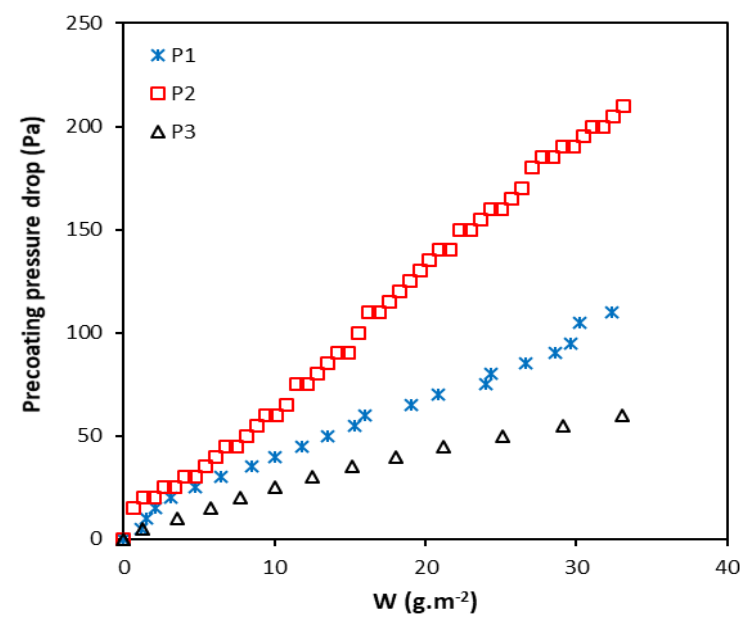

Figure 6: Precoating pressure drop evolution as a function of the deposited mass for a face velocity of $3 \mathrm{~cm} \cdot \mathrm{s}^{-1}$ using the three materials

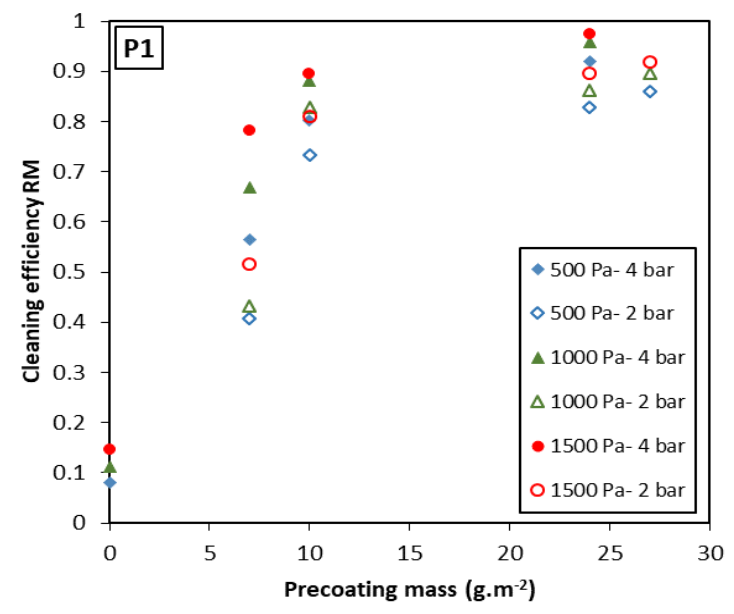

Figure 7: Variation of the cleaning efficiency with the precoating mass, the maximum pressure drop before cleaning and the cleaning air pressure using P1 for a face velocity of $3 \mathrm{~cm} . \mathrm{s}^{-1}$ 


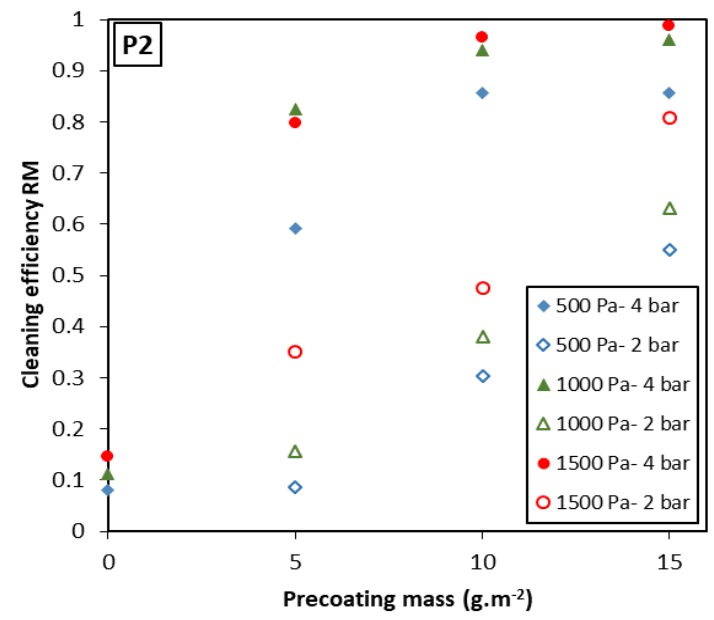

Figure 8: Variation of the cleaning efficiency with the precoating mass, the maximum pressure drop before cleaning and the cleaning air pressure using $\mathrm{P} 2$ for a face velocity of $3 \mathrm{~cm} . \mathrm{s}^{-1}$

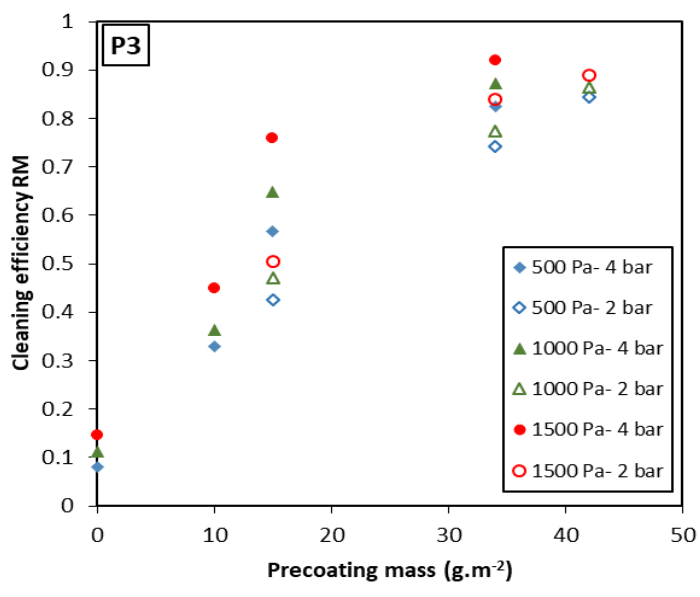

Figure 9: Variation of the cleaning efficiency with the precoating mass, the maximum pressure drop before cleaning and the cleaning air pressure using P3 for a face velocity of $3 \mathrm{~cm} . \mathrm{s}^{-1}$

\subsection{Clogging/unclogging cycles}

In a first step, a one-time precoating was tested, i.e. applying precoating only at the first cycle. The experiment was conducted because after the cleaning of filters with precoating, an extremely thin layer of precoating (that do not constitute a filter cake) always keeps adhering to the surface. This raised the question on the ability of this thin layer on ensuring a stable filtration process. Figure 10 compares the results obtained for a one-time precoating and without precoating. The maximum pressure drop before cleaning was set at $1500 \mathrm{~Pa}$ and the deposited mass of precoating P1 at $24 \mathrm{~g} \cdot \mathrm{m}^{-2}$. The cleaning was performed with a cleaning air pressure of 4 bar and without stopping the metallic nanoparticles generation. Applying precoating for just one time was not enough as the third cycle evolution was very rapid and the residual pressure drop, which represents the registered pressure drop after cleaning, kept increasing indicating an inefficient patchy cleaning. As found previously, a thin layer cannot protect the filter surface from the nanoparticles. Nevertheless, the results are still better than those without precoating, where the first cleaning was clearly inefficient and the residual pressure drop values are extremely high.

Figure 11 presents the evolution of the filtration process for an application of precoating P1 after each cleaning with a deposited mass of $24 \mathrm{~g} \cdot \mathrm{m}^{-2}$. The cleaning was performed once reaching a maximum pressure drop of $1500 \mathrm{~Pa}$, then the precoating was reapplied while stopping the metallic nanoparticles generation. The applied layer thickness was controlled by the pressure drop. The experiments were not realized with the other precoating materials as it would give similar trends, the difference is in the necessary mass as established by the former experiments. Results show that with a precoating application after each cleaning the filtration process became highly stable, as an efficient cleaning is performed at each cycle. The slight fluctuations of the precoating application time can be explained by occasional instabilities of the precoating generation. The same experiment was conducted with a face velocity of $8 \mathrm{~cm} \cdot \mathrm{s}^{-1}$ and a maximum pressure drop before cleaning of $2000 \mathrm{~Pa}$. Operating filtration systems at a high velocity can be appealing in the industry, as it allows reducing the filtration surface which in its turn helps saving costs. This will be associated with a decrease of the filter performances, in terms of initial collection efficiency and dust holding capacity, that would be compensated by the precoating layer. However, the velocity increase could bear modification to the cake porosity, it is well established in the literature that the porosity is a decreasing function of the velocity (Dennis and Klemm, 1980) (Silva et al., 1999), (Saleem et al., 2012), meaning further increase of the pressure drop. Moreover, because the governing collection mechanism for nanoparticles is Brownian diffusion, increasing the velocity will result in a lower collection efficiency which leads to penetration of ultrafine particles to the surface. This may require increasing the precoating thickness to ensure a better protection of the filter from the ultrafine particles. The results (figure 12) are presented as the ratio of the residual pressure drop $(\Delta \mathrm{Pr})$ to the face velocity $(U)$ as a function of the cycle number, and as the ratio of the cycle time to the first cycle duration as a 
function of the cycle number. Comparing to the results obtained at face velocity of $3 \mathrm{~cm} . \mathrm{s}^{-1}$, the stability of the filtration process does not seem to be affected by the velocity increase. A slight increase of the residual pressure drop is registered after each cycle, indicating a penetration of the nanoparticles trough the precoat layer during cycles. This accumulation of particles over cycles on the filter surface also causes a decrease of the cycle duration. This is interesting, as it shows the possibility of raising the face velocity without affecting the filter regeneration. Nevertheless, the choice of the precoating mass must also take into account the initial filtration performances.
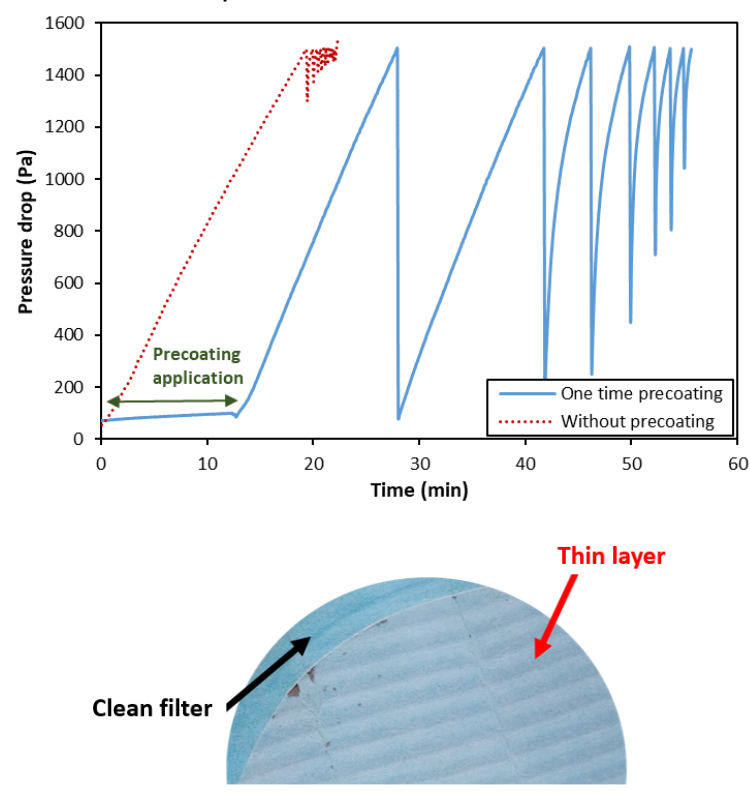

Figure 10: Results of a one-time precoating application with P1 material for a face velocity of 3 $\mathrm{cm} . \mathrm{s}^{-1}$ and an air cleaning pressure of 4 bar.

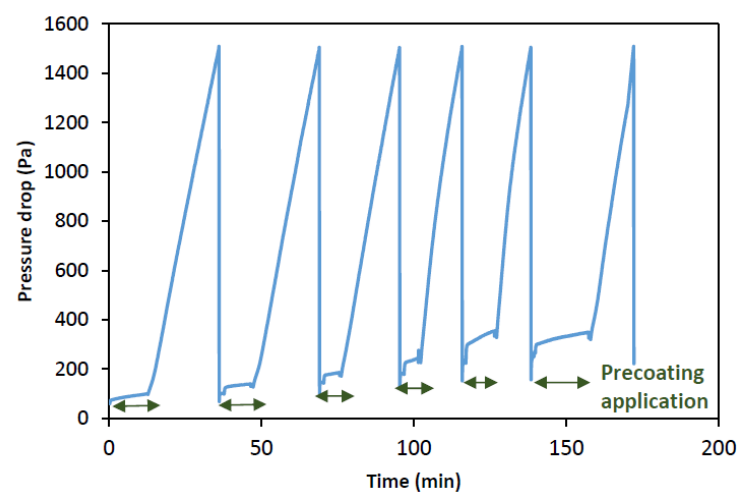

Figure 11: Clogging/unclogging cycles with application of precoating using $\mathrm{P} 1$ material after each cleaning for a face velocity of $3 \mathrm{~cm} . \mathrm{s}^{-1}$ and an air cleaning pressure of 4 bar.
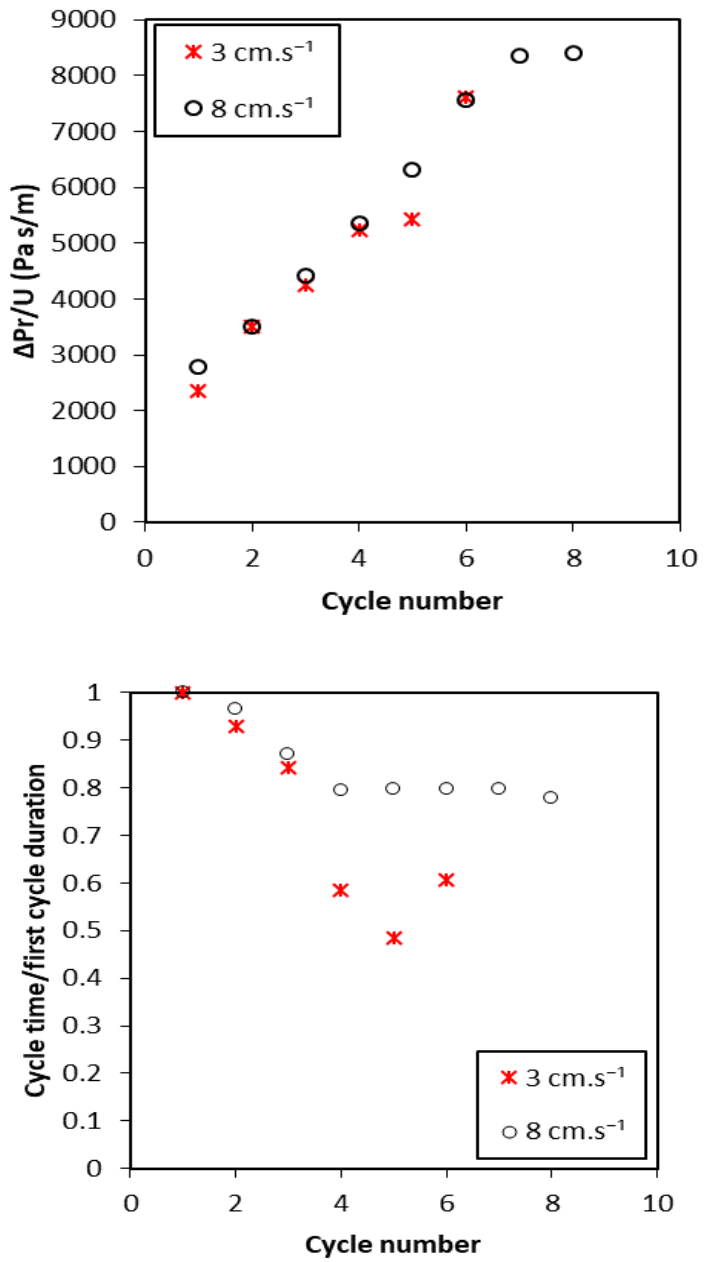

Figure 12: Comparing the cleaning performances during cycles for face velocities of 3 and $8 \mathrm{~cm} \cdot \mathrm{s}^{-1}$

\section{Conclusion}

This paper investigated the use of precoating for enhancing the cleaning of flat filters challenged with sticky metallic nanoparticles. Different materials were tested, among them a metallic powder recovered at the hopper of a cyclone that is available on site. Results revealed the beneficial effects of the preocating layer on the filtration efficiency and the dust holding capacity. Indeed, the precoating is an additional filtering medium that allows capturing nanoparticles. It also allows to considerably improve the cleaning efficiency of the filter media. The regeneration efficiency went from $15 \%$ without precoating to $90 \%$ with precoating. Increasing the collected mass of nanoparticles permits improving the cleaning efficiency. Moreover, experimental data showed that a thin layer of precoating is not enough to protect the filter surface from irreversible clogging. The precoating layer must be thick enough to prevent the nanoparticles from penetrating and reaching the 
filter surface. This minimum mass of precoating to be applied depends on the used material as the precoat layer structure and porosity differ with the particles shape, density and size.

With the use of precoating, the effect of operating conditions was studied by reducing the cleaning air pressure and increasing the filtration velocity. This could make the precoating application more attractive, as it could help reduce costs. Filtration cycles results showed that the precoating helped stabilize the filtration process and that renewing the precoating layer after each cleaning is essential to maintain good performances.

The results of this study lay the basis for a future work concerning the application of precoating on pleated filter cartridges. A precoating mass in the range of 20 - $40 \mathrm{~g} . \mathrm{m}^{-2}$ will be considered and the maximum pressure drop before cleaning will be set at the highest value possible. Reducing the cleaning air pressure will also be tested. The characteristics of the pleated filter cartridge are expected to be of influence, mainly the pleat number. Furthermore, a thorough characterisation of the precoating structure is needed, as it could help predict the precoating layer performances and provide guidelines for the application of precoating. Results did show that a precoating layer with high filtration efficiency seems to be appropriate to protect the filter surface.

\section{REFERENCES}

Bémer, D., Régnier, R., Morele, Y., Grippari, F., AppertCollin, J.C., Thomas, D., 2013. Study of clogging and cleaning cycles of a pleated cartridge filter used in a thermal spraying process to filter ultrafine particles. Powder Technol. 234, 16. https://doi.org/10.1016/j.powtec.2012.09.035

Charvet, A., Pacault, S., Bourrous, S., Thomas, D., 2018. Association of fibrous filters for aerosol filtration in predominant Brownian diffusion conditions. Sep. Purif. Technol. 207, 420-426. https://doi.org/10.1016/j.seppur.2018.06.045

Clift, R., Seville, J.P.K., 1993. Gas Cleaning at High Temperatures, Springer. Springer Netherlands, Dordrecht. https://doi.org/10.1007/978-94-011-2172-9

Dennis, R., Klemm, H.A., 1980. Modeling Concepts for Pulse Jet Filtration. J. Air Pollut. Control Assoc. 30, 38-43. https://doi.org/10.1080/00022470.1980.10465912

Förster, H., Thajudeen, T., Funk, C., Peukert, W., 2016. Separation of nanoparticles: Filtration and scavenging from waste incineration plants. Waste Manag. 52, 346-352. https://doi.org/10.1016/j.wasman.2016.03.050

Hajar, S., Rashid, M., Nurnadia, A., Ammar, M.R., 2015. The characteristics of a formulated filter aids for fabric filters. Powder Technol. 283, 315-320. https://doi.org/10.1016/j.powtec.2015.05.035

Khirouni, N., Charvet, A., Thomas, D., Bémer, D., 2020. Regeneration of dust filters challenged with metallic nanoparticles: Influence of atmospheric aging. Process Saf.
Environ.

Prot.

138

$1-8$.

https://doi.org/10.1016/j.psep.2020.02.040

Lo, L.M., Chen, D.R., Pui, D.Y.H., 2010. Experimental study of pleated fabric cartridges in a pulse-jet cleaned dust collector. Powder Technol. 197, 141-149. https://doi.org/10.1016/j.powtec.2009.09.007

Peukert, W., Wadenpohl, C., 2001. Industrial separation of fine particles with difficult dust properties. Powder Technol. 118, 136-148. https://doi.org/10.1016/S00325910(01)00304-7

Saleem, M., Krammer, G., Tahir, M.S., 2012. The effect of operating conditions on resistance parameters of filter media and limestone dust cake for uniformly loaded needle felts in a pilot scale test facility at ambient conditions. Powder Technol. 228, 100-107. https://doi.org/10.1016/j.powtec.2012.05.003

Schiller, S., Schmid, H.J., 2015. Highly efficient filtration of ultrafine dust in baghouse filters using precoat materials. Powder Technol. 279, 96-105. https://doi.org/10.1016/j.powtec.2015.03.048

Schiller, S., Schmid, H.J., 2014. Ultrafine dust filtration using precoat materials considering the influence of filter media. Chem. Eng. Technol. 37, 1009-1020. https://doi.org/10.1002/ceat.201300856

Schmidt, E., 2004. Raw Gas Conditioning for Improving Particle Separation. Fluid Partice Sep. 16, 193-199.

Schmidt, E., Löffler, F., 1990. Collection of fine adhesive and cohesive dusts with pluse-jet fabric filers. Chem. Eng. Technol. 13, 402-410. https://doi.org/10.1002/ceat.270130155

Schmidt, E., Pilz, T., 1996. Raw gas conditioning and other additional techniques for improving surface filter performance. Filtr. Sep. 33, 409-415. https://doi.org/10.1016/S0015-1882(97)84301-7

Sievert, J., Löffler, F., 1987. Dust cake release from non woven fabrics. Filtr. Sep. 424-427.

Silva, C.R.N., Negrini, V.S., Aguiar, M.L., Coury, J.R., 1999. Influence of gas velocity on cake formation and detachment. Powder Technol. 101, 165-172. https://doi.org/10.1016/S0032-5910(98)00168-5

Zhang, Q., Schmidt, E., 2012. Effects of Post-Coating By Generating a Thin Secondary Particle Layer. 11th World Filtr. Congr. 